\title{
Residual correction WLS based on Indoor Location Algorithm Considering Arrival Angle and Phase Difference Parameter Estimation
}

\author{
Lu An ${ }^{1, a, *}$, Li Hui ${ }^{2, b}$, Gong Yingkun ${ }^{3, c}$ Yang Li $^{4, d}$ \\ Chongqing Inspection Institute for Metrology and Quality,Chongqing401121, China \\ a'luan527@163.com, blihui@sina.com, gyingkun@126.com, dyabgli@sohu.com
}

Keywords: Non-line of sight, Arrival phase difference, Residual correction.

\begin{abstract}
Aiming at the problem of signal multipath and non-line-of-sight propagation in the indoor positioning environment, this paper proposes a residual correction and weighted least squares algorithm. Firstly, the multipath propagation signal is identified by the antenna array and the propagation path. Then, the arrival angle and the propagation distance of the two signals is obtained by the estimation algorithm. The simulation results show that the proposed algorithm can improve the accuracy of indoor positioning, and it has certain advantages in positioning time compared with other indoor localization algorithms.
\end{abstract}

\section{Introduction}

With the increasing space and complexity of people's indoor activities, the demand for location services is becoming more and more obvious. In the indoor environment, due to the complex indoor environment and non-line-of-sight transmission caused by the obstruction, it makes the indoor positioning technology has a very big challenge.

In view of the characteristics of indoor positioning, the current indoor positioning methods mainly focus on reducing the cost of positioning services and improving positioning accuracy.In the complex indoor propagation environment, non-direct transmission of the signal is the main feature of the indoor channel ${ }^{[1]-[2]}$.In order to solve it, He Jie et al. proposed a least squares indoor three-dimensional localization algorithm based on the distance-value optimization ${ }^{[3]-[4]}$. Li Huaming et al. proposed a kind of algorithm based on kernel function feature extraction for the unique redundancy and noise problem under realistic wireless network environment ${ }^{[5]}$. That algorithm not only improves the positioning accuracy, but also reduces the average positioning error. But the prerequisite is to require the object to be measured still, and the situation of positioning of mobile targets remains to be further improved.

In this paper, by using the joint parameter estimation method based on the arrival angle and the arrival phase difference, the joint weighted least squares and the residual correction algorithm, the position of the object can be calculated.

\section{Theoretical analysis}

\subsection{Antenna array signal model and arrival angle estimation}

Considering the N-equidistant uniform linear array with a half-wave length of the array spacing, there are $\mathrm{M}$ narrow-band points in the far-field space with the incident angle $: \theta_{i}(i=1,2, \cdots, M)$.The array receives data for:

$$
X(t)=\left[x_{1}(t), x_{2}(t), \cdots x_{N}(t)\right]^{T}=A \cdot S(t)+N(t)
$$

where: array popularation: $\quad A=\left[a\left(\theta_{1}\right), a\left(\theta_{2}\right), \cdots a\left(\theta_{M}\right)\right]$, Oriented vector: 
$a\left(\theta_{i}\right)=\left[1, e^{j \cdot \beta_{i}}, \cdots e^{j \cdot(N-1) \beta_{i}}\right]^{T}$,Source wave number: ${ }^{\beta_{i}=\frac{2 \pi f}{\lambda} \cdot \sin \theta_{i}}$ ( $\lambda$ represents the wavelength, $d$ stands for source spacing. $S(t)=\left[s_{1}(t), s_{2}(t), \cdots s_{M}(t)\right]^{T}, s_{1}(t)$ represents a complex envelope of the i-th source; $N(t)=\left[n_{1}(t), n_{2}(t), \cdots n_{N}(t)\right]^{T}$ represents a Gaussian white noise with an independent mean distribution of zero and a variance of $\sigma^{2}$, which is not related to the signal.

The spatial smoothing algorithm defines the array of output covariance matrices:

$$
R=E\left[X(t) \cdot X(t)^{H}\right]=A \cdot R_{s} \cdot A^{H}+\sigma^{2} \cdot I_{N}
$$

among them, $R_{s}=E\left[S(t) \cdot S(t)^{H}\right]$ is the source covariance matrix; $\sigma^{2}$ is the noise power; $I_{N}$ is the identity matrix of the same dimension as R.

Spatial Smoothing Algorithm Formula:

$$
\tilde{R}=F_{1}\left(a_{i}, R_{i i}\right)+F_{2}\left(\beta_{i j}, R_{i j}\right) \text {, }
$$

According to the output covariance matrix $R$ and the smoothing algorithm formula $\tilde{R}$, the original array is divided into several subgroups which are partially overlapped with each other, and summarize the self and crossover covariance matrices between subgroups. By using the relationship between the sub array vector, the rank of sum of the covariance matrix is equal to the number of the source, and the target parameter estimation is realized by the conventional array signal processing algorithm. The two path arrival angles of the strongest signal are obtained, which are denoted as $\theta_{1}^{\prime}$ and $\theta_{2}^{\prime}$ respectively.

\subsection{Path estimation of arrival phase difference}

The array antenna is used to distinguish the multipath signal, and two strongest signal paths are selected according to the energy of the received signal, and the phase information of the two signal paths is obtained from the antenna.

The terminal A node transmits the radio frequency signal with the frequency $f_{1}$ and the initial phase $\phi_{1 t}$ to the array antenna B node. The node B measures the signal phase $\phi_{1 t}$ with the frequency f1 according to the phase X2 of the local co-frequency signal.And then the two nodes will convert the frequency used to ranging to frequency $f_{1}$, the radio signal with frequency $f_{2}$ is sent from the node A to the node B. The measured phase in the node B is $\phi_{2 r}$, as shown in Figure.1.

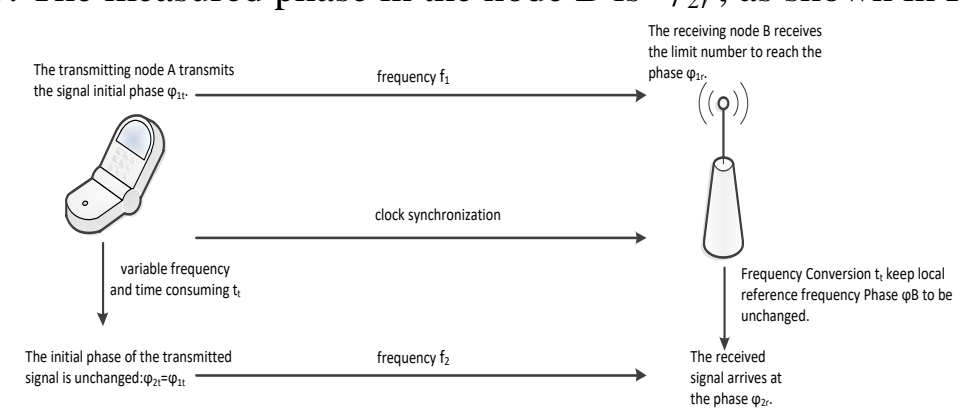

Figure.1 Principle of distance measurement for arrival phase difference

The phase difference between the two measurements is denoted by $\Delta \phi$ :

$\Delta \varphi=\left(\varphi_{2}-\varphi_{1}\right)=\left(\varphi_{2 r}-\varphi_{2 t}\right)-\left(\varphi_{1 r}-\varphi_{1 t}\right)=\left(\varphi_{2 r}-\varphi_{1 r}\right)-\left(\varphi_{2 t}-\varphi_{1 t}\right)$

Where: $\varphi_{1} 、 \varphi_{2}$ respectively, said the first and second phase measurement, $\mathrm{B}$ measured signal arrival phase and the difference between the initial phase of the signal. $\varphi_{1 t}$ and $\varphi_{2 t}$ for the same launch node, at different sub-frequency launch time to lock the initial phase of the transmitted signal, that is, $\varphi_{2 t}=\varphi_{1 t}$, So the measured value of the phase change measured at the receiving end, that is, $\Delta \varphi=\varphi_{2 r}-\varphi_{2 t}$, at the same time as the initial phase of the transmitter, $\Delta \varphi$ is only related to the measured phase of the receiver, avoiding the effect of phase discontinuity at both ends of $\mathrm{A}$ and $B{ }^{[7]}$.Therefore, under the ideal environmental conditions (no other RF signal interference, no multipath effect, etc.), The factors that cause the measurement phase change are only the frequency of the RF signal, and further calculated: 


$$
\Delta \varphi=\left(\varphi_{2}-\varphi_{1}\right)=\varphi_{2 r}-\varphi_{1 r}=2 \pi\left(\frac{d}{\lambda_{2}}-\left[\frac{d}{\lambda_{2}}\right]\right)-2 \pi\left(\frac{d}{\lambda_{1}}-\left[\frac{d}{\lambda_{1}}\right]\right)
$$

Where: $\lambda_{1}$ and $\lambda_{2}$ denote the wavelengths of the electromagnetic waves with frequencies $f_{1}$ and $f_{2}$, respectively, and $\mathrm{d}$ is the distance between nodes $\mathrm{A}$ and $\mathrm{B}$.

$d / \lambda$ represents the number of wavelengths in the interval between nodes. In the case of electromagnetic waves with firing frequencies of $f_{1}$ and $f_{2}$, the interval is very short, in microseconds, the distance of the nodes moving is much less than one wavelength, so it can be assumed that the numbers of integer multiple wavelengths in the two phases are the same ${ }^{[8-9]}$.Equation (5) can be simplified as:

$$
\Delta \varphi=\varphi_{2}-\varphi_{1}=2 \pi\left(\frac{d}{\lambda_{2}}-\frac{d}{\lambda_{1}}\right)
$$

Where $\lambda$ is the wavelength of the electromagnetic wave of the frequency used. The distance $d$ between nodes can be expressed as follows:

$$
d=\frac{c \cdot \Delta \varphi}{2 \pi\left(f_{2}-f_{1}\right)}
$$

In order to reduce the influence of channel fading on the measurement results and improve the accuracy of the calculation results, the system takes $2210 \mathrm{MHZ}$ as the starting frequency, add $2 \mathrm{MHz}$ for each measurement and $2250 \mathrm{MHZ}$ as the termination frequency, and the phase of each frequency point is measured. During the measurement process, the distance between nodes is very small, and the mean value is calculated after each distance measurement. The distance between the two nodes is used:

$$
d=\frac{\sum_{N} d_{i}}{N}=\frac{c}{2 \pi} \cdot \frac{\sum_{N} \Delta \varphi_{i}}{N \Delta \varphi}
$$

Where $\mathrm{i}$ is the $\mathrm{i}$-th distance, and the distance from the two paths is obtained from Equation (8), denoted by $d_{1}$ and $d_{2}$.

\subsection{Non-line-of-sight path and line-of-sight path transformation}

According to the location of the total obstacle in the positioning scene, the virtual base station is constructed, and the non-line-of-sight path can be transformed into the line-of-sight path. As shown in Fig.2 and Fig.3, $x_{b}$ is the position of the array antenna, $x_{t}$ is the position of the object to be positioned, and $X_{v}$ is the virtual base station established according to the position established by the obstacle 1 . Between the virtual base station and the object to be positioned, a line-of-sight path can be obtained.

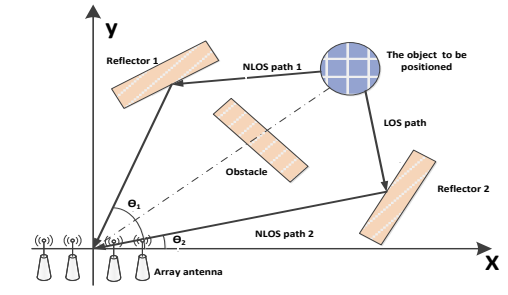

Figure.2 Scene-simulation diagram for positioning

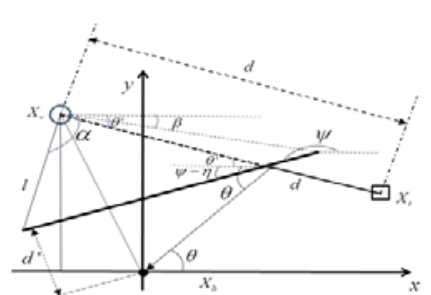

Figure.3 Simulation chart of NLS path to LS path

\subsection{Determine the position coordinate of the object to be determined}

According to the angle information and distance information obtained in Sections 2.1 and 2.2, the virtual base station established in Section 2.3, the weighted least squares algorithm is used to calculate the position of the object to be positioned, as follows:

The distance $\mathrm{d}$ and the angle $\theta$ provided with the $\mathrm{N}$ signal paths are estimated by the solid base station, $X_{k}=\left[x_{k}, y_{k}\right]^{T}, k=1,2, \cdots, N, 2,$. . . , is a virtual base station corresponding to the $\mathrm{k}$-th signal path, $d_{k} \mathrm{D}$ is the path length of the k-th signal path estimated by PDOA, and $d_{k} \leq d_{k+1}, \theta_{k}$ is the angle of arrival of the k-th signal path; Use $X_{k}=[x, y]^{T}$ to represent the position of the object to be positioned, there are:

$$
-2 x_{i} x-2 y_{i} y+x^{2}+y^{2}=d_{i}^{2}-x_{i}^{2}-y_{i}^{2}
$$


$x \sin \left(\theta_{i}\right)-y \cos \left(\theta_{i}\right)=x_{i} \sin \left(\theta_{i}\right)+y_{i} \cos \left(\theta_{i}\right)$

Equation (7) and (8) are written in the form of a matrix, and $G Z=H$ can be obtained, where:

$$
G=\left[\begin{array}{ccc}
-2 x_{1} & -2 y_{1} & 1 \\
\vdots & \vdots & \vdots \\
-2 x_{N} & -2 y_{N} & 1 \\
\sin \left(\theta_{1}\right) & \cos \left(\theta_{1}\right) & 0 \\
\vdots & \vdots & \vdots \\
\sin \left(\theta_{N}\right) & \cos \left(\theta_{N}\right) & 0
\end{array}\right] \quad H=\left[\begin{array}{c}
d_{1}^{2}-\left(x_{1}^{2}+y_{1}^{2}\right) \\
\vdots \\
d_{N}^{2}-\left(x_{N}^{2}+y_{N}^{2}\right) \\
x_{1} \sin \left(\theta_{1}\right)-y_{1} \cos \left(\theta_{1}\right) \\
\vdots \\
x_{N} \sin \left(\theta_{N}\right)-y_{N} \cos \left(\theta_{N}\right)
\end{array}\right], Z=\left[x, y, x^{2}+y^{2}\right]^{T}
$$

The weighted least squares algorithm is used to calculate the position of the object to be positioned, and the initial position coordinate $\left(x^{\prime}, y^{\prime}\right)$ of the object to be positioned is obtained:

$$
Z_{1}=\left[x^{\prime}, y^{\prime}, x^{\prime 2}+y^{\prime 2}\right]^{T}=\left(G^{T} W G\right)^{-1} G^{T} W H
$$

Where, $W=\frac{1}{B} \operatorname{diag}\left\{d_{N, \ldots}^{2} d_{1}^{2}, d_{N, \ldots}^{2} d_{1}^{2}\right\}$, $\operatorname{diag}\{\cdot\}$ represents a diagonal matrix, $B=\sum_{i=1}^{N} d_{i}^{2}$.

Suppose $d_{w k}=\sqrt{\left(x^{\prime}-x_{k}\right)^{2}+\left(y^{\prime}-y_{k}\right)^{2}}, k=1,2, \cdots N$, The residual weighting algorithm is used to optimize Equation (10) to obtain a more accurate position of the object to be positioned:

$$
X=[x, y]^{T}
$$

Where: $\quad x=\frac{\left(d_{w N}-d_{N}\right)^{2} x_{1}^{\prime}+\ldots\left(d_{w 1}-d_{1}\right)^{2} x_{N}^{\prime}}{\left(d_{w N}-d_{N}\right)^{2}+\ldots+\left(d_{w 1}-d_{1}\right)^{2}}, y=\frac{\left(d_{w N}-d_{N}\right)^{2} y_{1}^{\prime}+\ldots\left(d_{w 1}-d_{1}\right)^{2} y_{N}^{\prime}}{\left(d_{w N}-d_{N}\right)^{2}+\ldots+\left(d_{w 1}-d_{1}\right)^{2}}$

$X_{i}^{\prime}=\left[x_{i}^{\prime}+y_{i}^{\prime}\right]^{T}, i=1,2, \ldots, N$ is the position of the object to be positioned derived from the i-th signal path.

\section{Simulation results and analysis}

In order to verify the validity of the algorithm (UAD-WLS), the test environment is on the first floor of the Quality Inspection Institute. Compared with the WLS and LS-RW algorithms, the location error and average location error are used as evaluation parameters, and the formula is:

$$
\operatorname{err}_{i}=\sqrt{\left(x_{i}-x_{i}^{\prime}\right)^{2}+\left(y_{i}-y_{i}^{\prime}\right)^{2}}, \overline{e r r}=\frac{1}{n} \sum_{i=1}^{n} \operatorname{err}_{i}
$$

Where $\mathrm{n}$ is the number of test points.

Using WLS, LS-RW and WLS-RW positioning experiments, positioning results shown in Figure.4, we can get the following conclusions:

1)Compared with LS and LS-RW, WLS-RW can find more reasonable parameter values, and can better fit the mapping between feature vector and position information. The positioning accuracy is increased correspondingly, while positioning results become more reliable.

2)As can be seen from Figure.5, the probability of WLS-RW is about $20 \%$. In the range of $0-0.5$, it is clear that the probability of WLS-RW is equivalent to that of LS-RW. However, when the positioning error is more than $0.5 \mathrm{~m}$, the probability of WLS-RW is obviously larger than that of LS-RW and the positioning accuracy is less than $0.5 \mathrm{~m}$ with a probability of $80 \%$, which greatly reduces the system cost and reduces the difficulty of deployment, so the use of this proposed algorithm can achieve accurate indoor positioning.

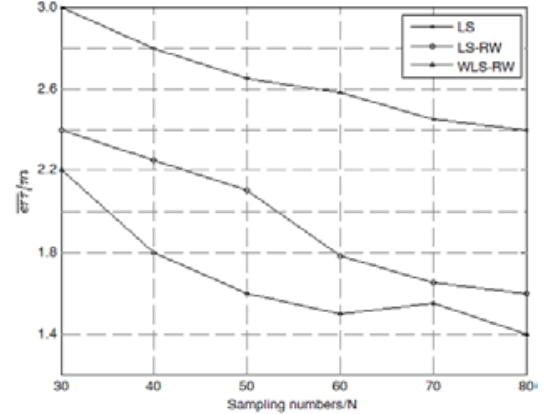

Figure.4 Comparison chart for APE

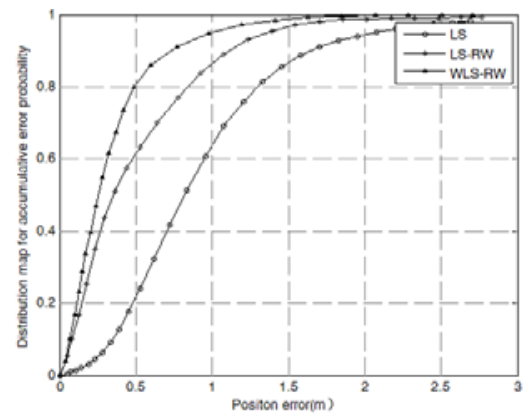

Figure.5 Distribution of probability accumulation for PE

In order to test the real-time performance of three algorithms, the experimental hardware 
platform adopts lenovo Y700 workstation, and the software system adopts Win7 operating system + Matlab R2015b. On this test platform, the running time is counted, as shown in Table 1. It can be seen from it that LS has the shortest run time and the best real-time performance, but it is not too practical to set the parameters, and its positioning accuracy is too low to be applied to practice. WLS-RW is running less than LS-RW, mainly because the optimization speed of WLS-RW algorithm is superior to LS-RW, and the training time is shorter, and the positioning real-time is better, and thus it is more suitable for indoor positioning.

Table 1 Contrast for running time

\begin{tabular}{|c|c|c|c|c|c|}
\hline $\begin{array}{c}\text { ntrmber of samples } \\
\text { algorithm }\end{array}$ & 30 & 40 & 50 & 60 & 70 \\
\hline LS & 48.91 & 60.26 & 67.56 & 68.38 & 81.35 \\
\hline LS-RW & 195.33 & 237.51 & 247.13 & 279.42 & 290.28 \\
\hline WLS-RW & 149.18 & 151.96 & 152.46 & 171.75 & 182.77 \\
\hline
\end{tabular}

\section{Conclusion}

In this paper, we propose a residual correction WLS antenna array indoor localization algorithm jointing arrival angle and phase difference parameter estimation. The algorithm uses the antenna array to obtain the two most powerful signal propagation path and its phase information. Based on the arrival angle and phase difference estimation method, the algorithm combines the weighted least squares and residual correction algorithms to calculate the position of the object to be positioned. At the same time, the virtual base station is used to transform the non-line-of-sight path into the line-of-sight path to improve the influence of its indoor position and thus the indoor positioning accuracy. The results show that the accuracy of WLS-RW positioning is higher than other algorithms, and the running time is more advantageous, and it can give users a better indoor positioning effect.

\section{References}

[1]Li Hualiang, Qian Zhihong,Tian Hongliang, Research on indoor localization algorithm based on kernel principal component analysis[J].Journal of Communications,2017:38(1):218-225.

[2]Yuan Lifen,Zhang Yue,et al.An improved LANDMARC indoor localization algorithm based on CKF[J].Journal of Electronic Measurement\&Instrumentation, 2017:31(5):739-744.

[3]He Jie,Wu Deyang,et al.A 3-D Indoor Localization Algorithm Using Distance Optimization [J].Journal of Beijing University of Posts and Telecommunications,2017:38(3):326-333.

[4]Wang Peng,Zhang Xiaotong,et al. Indoor near field ranging algorithmbased on adaptive time delay estimation[J].Chinese Journal of Computers, 2016,39(32):1-19.

[5]Li Zeyuan.Constrained weighted least squares location algorithm using receivedsignal strength measurements[J].China Communications,2016,13(4):81-88.

[6]ZHANG H,BI G,CAI Y,et al.DOA estimation of closely-spaced and spectrally-overlapped sources using a STFT-based MUSIC algorithm[J].Digital Signal Processing,2016,52:25-34.

[7]Zhou Jin Li Wei,et al.Indoor positioning system based on KNN-SVM algorithm[J].Journal of Huazhong University of Science and Technology (Natural ScienceEdition),2015,43(1):517-520.

[8]Liang SC,Liao LH,Lee YC.Localization algorithm based on improved weightedcentroid in wireless sensor networks[J].Journal of Networks,2014,9(1):183-189.

[9]GHOFRANI S,AMIN M G,ZHANG Y D.High-resolution directionfinding of non-stationary signals using matching pursuit[J].Elsevier Signal Processing,2013,93(12):3466-3478. 